\title{
GROUPS WITH THE INFINITE NON-QUASICENTRAL NODAL SUBGROUP
}

We present some properties of the groups with the infinite non-quasicentral periodic nodal subgroup. Our main results are formulated in Theorem 1, 2 and Theorem 3, 4. 2000 Mathematics Subject Classification: 20F22, $20 F 25$

Keywords: A group, a subgroup, a commutator of a group, a locally graded group, p-quasicyclic group, a direct and semi-direct product of groups, an extension of a group.

The description of groups defined by the systems of their subgroups was first described in the papers of Chernikov and Kurosh (RN - groups, [1]). Chernikov dealt with an extension of the direct product of the finite number of the quasicyclic groups by the finite abelian group (ч - groups, [2]), with the infinite non-abelian groups whose arbitrary infinite subgroup is the normal subgroup of the whole group (INH - groups, [2]). Subbotin studied the groups $G$ in which every subgroup from commutator $\mathrm{G}^{\prime}$ is a normal subgroup of $\mathrm{G}$ (KI-groups, [3]). Tomanek, L. studied the IAN and the IANA groups, Definition 1, (IAN groups, [4]). This definition was given to the author by Chernikov. In this paper we describe IAN and IANA groups with the infinite non-quasicentral periodic nodal subgroup.

We use standard designations of terminology where: $M \times N$ is the direct product of the groups $M, N ; \sum_{i \in I} X i$ is the direct sum of the additive groups $X_{i}$ for all $i \in I, M \lambda N$ is the semidirect product of the groups $M, N ; \mathrm{M} . \mathrm{N}=[\mathrm{mn} \mid \mathrm{m} \in \mathrm{M}, \mathrm{n} \in \mathrm{N}\}$ is the product of the groups $M, N, G / A$ is the factor group of $G$ by $A,|G: N|$ is the index of the subgroup $N$ in a group $G$; $\langle a\rangle$ is the cyclic group generated by the element $a$; $\langle a, b, c\rangle$ is the group generated by the elements $a, b, c ; H \leq \mathrm{G}$ where $H$ is the subgroup of $G ; H \unlhd G, H$ is normal in $G ;[a, b]=a^{-1} b^{-1} a b$ is the commutator of the elements $a, b \in G ; G^{\prime}=[G, G]$ generated by all commutators of the elements $a, b \in G$ is the commutator of $G$; [5] $Z\left(p^{\infty}\right)=\left\{x \mid x^{p^{n}}=1, n=1,2, \ldots\right\} \quad$ is the $p$-quasicyclic group; $C_{G}(A)$ is the centralizer of the subgroup $A$ in $G ; C(G)$ is the centre of the group $G$; $G \cong H$ where the groups $G, H$ are isomorphic. The group $G$ is the $p$-group if each of its elements has an order with a power of some fixed prime $p$ [6]. The group $G$ is a locally graded group if every finitely generated nontrivial subgroup of $G$ contains a proper subgroup of finite index ([2] p.236). The group $\mathrm{G}$ is the solvable group if it includes series: $\left.G>G^{\prime}\right\rangle G^{(2)}>\ldots>G^{(n)}=\langle e\rangle$. The subgroup $A$ is a quasicentral of $G$ if every subgroup of $A$ is normal in $G$. The group $G$ is the extension of the group $H$ by a normal subgroup $N$ of $G$ if $G / N \cong H$. The extension of the finite direct product of the quasicyclic groups by the finite abelian group are $u$ - groups ([2]). The group $G$ is an almost quasicyclic group if $G$ is the extension of the quasicyclic group by the finite group. $Q_{8}=\left\langle x, y \mid x^{4}=y^{4}=1, x^{2}=y^{2}, y x=x^{-1} y\right\rangle$ is the quaternion group.

\section{Definition 1.}

An infinite non-abelian $\mathrm{G}$ is said to be the IAN group if there exists a subgroup $A$ of $G$ so that every infinite subgroup of $A$ and every infinite subgroup of $G$ containing $A$ is a normal subgroup of $G$. The group $G$ is the IANA group if $A$ is the abelian subgroup. The subgroup $A$ is called the nodal subgroup.

\section{Definition 2.}

An infinite non-abelian $G$ is the INH group if an arbitrary infinite subgroup of $G$ is the normal subgroup of $G$.

\section{Definition 3.}

The group $G$ is the Dedekind group if an arbitrary subgroup of $G$ is the normal subgroup of $G$. Non-abelian Dedekind group $G$ is called the Hamiltonian group.

\section{Proposition 1. [[2], T. 6.10]}

The infinite Hamiltonian groups and the non-abelian nonHamiltonian groups that are the finite extensions of the quasicyclic

\footnotetext{
* ${ }^{1}$ Ludovit Tomanek, ${ }^{2}$ Anna Tomankova

${ }^{1}$ Department of Mathematics, Faculty of Humanities, University of Zilina, Slovakia

${ }^{2}$ Institute of Foreign Languages, University of Zilina, Slovakia

E-mail: ludovit.tomanek@fhv.uniza.sk
} 
subgroups by the finite abelian and the finite Hamiltonian groups form the class of the solvable INH groups.

\section{Proposition 2.[[7], T.12.5.4]}

The group $G$ is the Hamiltonian group if and only if the group $G=Q_{8} \times M \times N$ where $Q_{8}$ is the quaternion group, $M$ is an elementary abelian 2- group, $N$ is a periodic abelian group with no elements of the order 2.

\section{Lemma 1.}

Let $G$ be the IAN group with a nodal subgroup A. If a nodal subgroup A contains the element of the infinite order, then $A$ is the abelian quasicentral subgroup of group $G$.

Proof. If the group $A$ contains the element $x$ of the infinite order, then according to Definition 2, the group $A$ is the $I N H$ group. According to Proposition $1 A$ is the abelian group. Let $B$ be an arbitrary subgroup of the group $A$. We shall show that $B \unlhd G$. If $B$ is an infinite subgroup of $G, B$ is admittedly a normal subgroup of $G$.

Let $B$ be a finite subgroup of $G$. If $A$ is the abelian group containing the element $\mathrm{x}$ of the infinite order, then $B\langle x\rangle \cong B \times\langle x\rangle$. Pursuant to Definition $1(B \times\langle x>) \unlhd G$ which implies $B \unlhd G$. Thus $A$ is the abelian quasicentral subgroup of the group $\mathrm{G}$.

\section{Lemma 2.}

If $G$ is the locally graded IAN group with the nodal subgroup $A$, there exists a subgroup of $A$ that is not a normal subgroup of $G$. Then $A$ is a finite group or $A$ is the extension of the quasicyclic subgroup by the finite Dedekind group.

Proof. Let $G$ be the $I A N$ group with a nodal subgroup $A$ and let $A_{1}<A$ where $A_{1}$ is not a normal subgroup of $G$. Admittedly, $A_{1} \neq\langle e\rangle$ is a finite subgroup of $G$. In agreement with Lemma $1 A$ is a periodic group. If $A$ is a finite group, then this lemma is valid. Let $A$ be an infinite periodic subgroup of $G$. We consider two possible cases; $A$ is not $u$-group, or $A$ is $u$-group.

Case 1. Let $A$ not be $u$-group. Then choose the subgroup $A_{2}$ of $A$ where $A_{2}=A_{3} \times A_{4}, A_{2} \cap A_{1}=\langle e\rangle$, and where $A_{3}, A_{4}$ are the infinite cyclic groups of $G$. By Definition $1 A_{3} \unlhd G, A_{3} \times A_{1} \triangleleft G$, $A_{4} \unlhd G, A_{4} \times A_{1} \unlhd$ G. Evidently $\left(A_{3} \times A_{1}\right) \cap\left(A_{4} \times A_{1}\right)=A_{1}$ and furthermore $A_{1} \unlhd G$, so it is a contradiction.

Case 2. Let A be $y$-group. Then put $A=R . B$ where $R$ is the direct product of the finite number of the quasicyclic groups, $R$ is at the same time a divisible group, and $B$ is the finite group where $B \neq<\mathrm{e}>$.Therefore, $A_{1}$ is not a normal subgroup of $G$; there exists a cyclic subgroup $\langle a\rangle$ of $A_{1}$ that is not normal in $G$ and where $R$ $\cap\langle a\rangle=\langle t\rangle$. Since $R$ is a divisible group, there exists a quasicyclic subgroup $R_{1}$ of $R$ and furthermore $R_{1}$ contains the subgroup $\left\langle t>\right.$.Put $R=R_{1} \times R_{2}$ where $R_{2}$ is an infinite subgroup of $A$ or $R_{2}=\langle e\rangle$. If $R_{2}$ is an infinite subgroup of $A$, then, by Definition 1
$R_{2} \unlhd \mathrm{G}$, furthermore $\left(R_{2} \lambda\langle a\rangle\right) \unlhd G, R_{1} \unlhd G,\left(R_{1}\langle a\rangle\right) \unlhd G$. Evidently $\left(R_{2} \lambda\langle a\rangle\right) \cap\left(R_{1}\langle a\rangle\right)=\langle a\rangle$ and $\langle a\rangle \unlhd G$. This is a contradiction.

Let $R_{2}=\langle e\rangle$, then $R=R_{1}$ is a quasicyclic group and moreover $A / R \cong B$ where $B$ is a finite Dedekind group. Thus $A$ is the extension of the quasicyclic subgroup by the finite Dedekind group.

\section{Theorem 1.}

If $G$ is a locally graded IAN group with a nodal subgroup A, then subgroup A belongs to one of the types:

1. A is a finite subgroup of $G$;

2. $A$ is an extension of the quasicyclic subgroup by a finite Dedekind group where $G$ 'is an infinite group;

3. $A$ is an infinite quasicentral periodic subgroup of $G$;

4. $A$ is a quasicentral non-periodic abelian subgroup of $G$.

Proof. If $A$ is not a quasicentral subgroup of $G$, then, based on Lemma 2, the subgroup $A$ belongs to one of types 1 or 2 of this theorem. If $A$ is a quasicentral subgroup of $G$, then by Lemma 1 the subgroup $A$ belongs to one of the types 3 or 4 of this theorem.

By Theorem 1 and according to the definition of IANA groups the next corollary follows.

\section{Corollary 1.}

If $G$ is a locally graded IANA group with a nodal subgroup A, then subgroup A belongs to one of the types:

1. A is a finite abelian subgroup of $G$;

2. $A=Z(p \infty) \times B$, where $B$ is a finite group;

3. $A$ is an infinite quasicentral periodic abelian subgroup of $G$;

4. $A$ is a quasicentral non-periodic abelian subgroup of $G$.

\section{Lemma 3.}

If $G$ is the locally graded group with the infinite periodic nodal subgroup A, then the subgroup A satisfies one of the following conditions:

1. A is the infinite periodic Dedekind quasicentral subgroup of the group $G$ where $G / A$ is the abelian group;

2. A is the infinite periodic Dedekind quasicentral subgroup of the group $G$ where $G / A$ is the Hamiltonian group and $G$ is a locally finite group;

3. $A$ is not the quasicentral subgroup of $G, A$ is an almost quasicyclic subgroup of $G$ where $G / A$ is the Dedekind group.

Proof. If $\mathrm{G}$ is the locally graded group with an infinite periodic nodal subgroup $A$, then, according to Theorem $1 A$ is the extension of the quasicyclic subgroup by the finite Dedekind group, or A is the quasicentral subgroup of the group $G$.

Let $A$ be the extension of the quasicyclic subgroup $B$ by the finite Dedekind group. If $B$ is an infinite subgroup of $G$ containing $A$, then $B \unlhd G$ and furthermore every quotient subgroup 
$B / A \unlhd \mathrm{G} / \mathrm{A}$. Since $G / A$ is the Dedekind group, $A$ then satisfies the $3^{\text {rd }}$ condition of this lemma.

If $A$ is a quasicentral subgroup of group $G$, then, analogous to the paragraph above, we can prove that $G / A$ is the Dedekind group. Admittedly, $G / A$ is the abelian or the Hamiltonian group. If $G / A$ is the abelian group, then $A$ satisfies the $1^{\text {st }}$ condition of this lemma.

Let G/A be the Hamiltonian group. By Proposition $2 G / A$ is a locally finite group. Thus an extension of a locally finite group by a locally finite group is a locally finite group, which implies that $G$ is a locally finite group and hence A satisfies the $2^{\text {nd }}$ condition of this lemma.

\section{Lemma 4.}

If $G$ is the locally graded IAN group with the infinite nodal subgroup A non-quasicentral of $G$, then $A$ is the extension of a quasicyclic group by the Dedekind group.

Proof. Let $G$ be the locally graded $I A N$ group with the infinite nodal subgroup $A$ non-quasicentral of $G$. According to Theorem $1 A$ is a periodic group, by Lemma $3 A$ is the extension of a quasicyclic group by the Dedekind group.

\section{Lemma 5.}

If $G$ is the group with a finite nodal subgroup $A$, then $G / A$ is the abelian group, or the group.

Proof. If $G / A$ is the abelian group, then this lemma is valid. Let $G / A$ be a non-abelian group and $B / A$ be an arbitrary infinite subgroup of $G / A$. There evidently exists $\mathrm{B} \unlhd \mathrm{G}$ and furthermore $B / A \unlhd G / A$. Thus $G / A$ is the $I N H$ group.

\section{Theorem 2.}

Let $G$ be the locally graded IAN group with a nodal subgroup A. The nodal subgroup $A$ of $G$ is a non-quasicentral of $G$ if and only if it satisfies one of the following conditions:

1. A is a finite non-quasicentral subgroup of $G$, the quotient group $G / A$ is the INH group with the abelian commutator or $G / A$ is the abelian group;

2. $A$ is an almost quasicyclic group which contains the finite subgroups that are not normal in $G,|A: A \cap G|<\infty$, and $G / A$ is the Dedekind group.

Proof. Let $G$ be the locally graded $I A N$ group with the infinite nodal subgroup $A$. Admittedly, the subgroup $A$ is a nonquasicentral of $G$. Referring to Lemma $2 A$ is a finite group, or $A$ is an almost quasicyclic group.

If $A$ is a finite group then, by Lemma $5, G / A$ is the abelian group or $G / A$ is a solvable $I N H$ group. According to Proposition 1 the commutator of a solvable $I N H$ group is the abelian group. Thus the subgroup $A$ satisfies the 1 st condition of this theorem.
Let $G / A$ be a solvable $I N H$ group. By the condition 3 of Lemma $3 G / A A$ is the Dedekind group. Based on this fact $A$ is an almost quasicyclic group and by Definition $1 A$ is a nonquasicentral of $G$. Suppose there exists a finite subgroup and $A$ is normal in $G$. Therefore $G / A$ is the Dedekind group, A is an almost quasicyclic group, thus $G^{\prime}$ is a subgroup of that almost quasicyclic subgroup $A$. $G^{\prime}$ of $G^{\prime}$. Let $G^{\prime}$ be a finite group and put $A=G^{\prime}$. Hence A satisfies either condition 1 or condition 2 of this theorem.

If $G^{\prime}$ is an infinite group, then $\left|A \cdot G^{\prime}: G^{\prime}\right|<\infty,\left|A: A G^{\prime}\right|<\infty$, too. Admittedly, $A$ satisfies the $2^{\text {nd }}$ condition of this theorem.

Conversely. Suppose the nodal subgroup $A$ satisfies either condition 1 or condition 2 of this theorem, then $G$ is the IAN group with the non-quasicentral nodal subgroup A.

By Theorem 2 and the definition of IANA groups the next corollary follows.

\section{Corollary 2.}

Let $G$ be a locally graded IANA group with a nodal subgroup A. The nodal subgroup $A$ of $G$ is a non-quasicentral of $G$ if and only if it satisfies one of the following conditions:

1. $A$ is the finite abelian non-quasicentral subgroup of $G$, the quotient group $G / A$ is the INH group with the abelian commutator or $G / A$ is the abelian group,

2. $A=Z(p \infty) \times D$ where $D$ is the finite abelian subgroup of $G, A$ contains finite subgroups that are not normal in $G$, and $G / A$ is the Dedekind group.

\section{Lemma 6.}

Let $G$ be the IAN group with the infinite non-quasicentral Dedekind nodal subgroup $A$. Then $A=Z(p \infty) \times D$ where $D$ is a finite Dedekind group, $p|| D \mid$, and there exists the element $a \in A$ so that the subgroup $\langle a\rangle$ is not normal $p$ - subgroup of $G$. For $p=2$ is $D^{\prime}=\langle e\rangle$.

Proof. Let $G$ be the $I A N$ group with the infinite nonquasicentral Dedekind nodal subgroup $A$. By Lemma $1 A$ is an almost quasicyclic group. Pursuant to Proposition $2 A=Z(p \infty) \times D$ where $D$ is a finite Dedekind group, $p=2$, and $D^{\prime}=\langle e\rangle$. We shall prove that A does not contain a normal $p$-group $\langle a\rangle$ of $G$.

Obviously, if every cyclic subgroup of $A$ is a normal subgroup of $G$, then $A$ is a quasicentral subgroup of $G$. Thus there exists a cyclic subgroup $\langle x\rangle$ of $A$ that is not normal in $G$, which implies that the $\langle a\rangle$ Sylow $q$ - subgroup of the group $\langle x\rangle$ is not normal in $G$. This verifies that $q=p$.

Let $q \neq p$ and $Z(p \infty) \cap\langle a\rangle=\langle e\rangle$. Then $Z(p \infty) .\langle a\rangle \cong Z(p \infty) \times\langle a\rangle$ where $Z(p \infty) \times\langle a\rangle$ is the normal subgroup of $G$. Evidently, $\langle a\rangle$ is the Sylow q-subgroup normal in $Z(p \infty) \times\langle a\rangle$ and $\langle a\rangle$ is normal in $G$. This is a contradiction, thus $q=p$.

If $A=Z(p \infty) \times D \geq Z(p \infty)\langle a\rangle$, then $Z(p \infty)\langle a\rangle=Z(p \infty) \times\langle b\rangle$, $\langle b\rangle=D \cap Z(p \infty) \times\langle a\rangle$. Because the subgroup $\langle a\rangle$ is the $p$ - subgroup 
normal in $G$ and furthermore $Z\left(p^{\infty}\right) \cap\langle a\rangle\langle\langle a\rangle,|b|>1,\langle b\rangle$ is a $p$-group, therefore $p|| D \mid$.

According to Lemma 6 and the Definition of IANA groups, the next corollary follows.

\section{Corollary 3.}

Let $G$ be the IANA group with the infinite non-quasicentral nodal subgroup $A$. Then $A=Z(p \infty) \times D$, where $D$ is a finite group, $p|| D \mid$, the subgroup $D$ contains an element a $A$ so that the $\langle a\rangle$ $p$ - subgroup is not normal in $G$.

\section{Theorem 3.}

The group $G$ is the locally graded IAN group with the infinite non-quasicentral nodal subgroup $A$ of $G$ if and only if a quotient group $G / A$ is the Dedekind group, $\left|A: A \cap G^{\prime}\right|<$ and the nodal subgroup $A$ is of one of the types:

1. $A=Z(p \infty) \times D$, where $D$ is the finite Dedekind group, $p=2, D^{\prime}=\langle e\rangle$, $p|| D \mid$, the subgroup A contains an element a such that $\langle a\rangle$ p-subgroup is not normal in $G$, and the quotient group $A / Z(p \infty)$ is the quasicentral in $G / Z(p \infty)$;

2. $A=Z(p \infty) \lambda D$, where $D$ is the finite Dedekind subgroup, the group $A$ does not contain the finite normal subgroup of $G$, and $A / Z(p \infty)$ is the quasicentral in $G / Z(p \infty)$;

3. $A=(Z(p \infty) \cdot B) \times D$, where $Z(p \infty) \cdot B$ is the non-abelian Syllow p-subgroup of $G, D$ is the infinite Dedekind group, $p=2$, $D^{\prime}=\langle e>, Z(p \infty) \leq C(G)$, the finite group $B$ has a normal series: $\left.Z(p \infty) \cap B=B^{\prime}=B_{0}<B_{1}<\ldots<B_{i}<\ldots<B_{n^{\prime}} n \geq 1, \quad B_{i}=B_{i-1} \lambda<b_{i}\right\rangle$, for all $i \geq 1,\left|b_{i}\right|>1$, and $A / Z(p \infty)$ is the quasicentral in $G / Z\left(p^{\infty}\right)$;

4. $A=(Z(p \infty)$. B $\left.) \lambda Q_{8}\right) \times D$, where $Z(p \infty) \cdot B \cdot Q_{8}$ is the Sylow 2-subgroup of $G, D$ is the finite abelian group, $Z(p \infty)<C(G)$, the finite group $B$ has a normal series:

$Z(p \infty) \cap B=\left\langle B^{\prime},\left[B, Q_{8}\right]\right\rangle=B_{0}<B_{1}<\ldots<B_{i}<\ldots<B_{n^{\prime}} \quad n \geq 1$, $B_{i}=B_{i-1} \lambda\left\langle b_{i}\right\rangle$, for all $i \geq 1,\left|b_{i}\right|=2,\left|B_{0}\right|=2$, and $A / Z(p \infty)$ is the quasicentral in $G / Z\left(p^{\infty}\right)$;

5. $A=\left(Z\left(2^{\infty}\right) \cdot B\right) .\langle d>) \times D$, where $Z\left(2^{\infty}\right) \cdot B \cdot\langle d>$ is the Sylow 2 - subgroup of $G, D$ is the finite abelian group, $Z\left(2^{\infty}\right) \leq C\left(Z\left(2^{\infty}\right) \cdot B\right)$, for each $c \in Z\left(2^{\infty}\right), d^{-1} c d=c^{-1}$, the finite group $B$ has a normal series: $Z\left(2^{\infty}\right) \cap B=\left\langle B^{\prime},[B,\langle d\rangle]\right\rangle=B_{0}\left\langle B_{1}<\right.$ $\left\langle B_{i}<\ldots<B_{n^{\prime}} n \geq 1, B_{i}=B_{i-1} \lambda\langle d\rangle\right.$, for all $i \geq 1,(Z(p \infty) . B) \cap\langle d\rangle=$ $Z\left(2^{\infty}\right) \cap\langle d\rangle \leq\left\langle c_{1}\right\rangle,\left|c_{1}\right|=2$, and $A / Z(p \infty)$ is the quasicentral in $G / Z(p \infty)$;

6. $A=\left(\left(\left(Z\left(2^{\infty}\right) \cdot B\right) \cdot\langle d>) \lambda Q_{8}\right) \times D\right.$, where $Z\left(2^{\infty}\right) \cdot B . Q_{8} \cdot\langle d\rangle$ is the Sylow 2- subgroup of $G, D$ is the finite abelian group, $Z\left(2^{\infty}\right)<C\left(Z\left(2^{\infty}\right) \cdot B . Q_{8}\right)$, for each $c \in Z\left(2^{\infty}\right), d^{-1} c d=c^{-1}$, the finite group $B$ has a normal series:

$Z\left(2^{\infty}\right) \lambda B^{\prime}=\left\langle B^{\prime},[B,\langle d\rangle],\left[B, Q_{8}\right],\left[\langle d\rangle, Q_{8}\right]\right\rangle=B_{0}\left\langle B_{1}<\ldots\right.$ $\left\langle B_{i}<\ldots<B_{n^{\prime}} n \geq 1, B_{i}=B_{i-1} \lambda\left\langle b_{i}\right\rangle\right.$, for all $i \geq 1,\left|b_{1}\right|=2,\left|B_{0}\right|=2$, $|d|=4,(Z(p \infty) \cdot B) \cap\langle d\rangle=Z\left(2^{\infty}\right) \cap\langle d\rangle$, and $A / Z\left(p^{\infty}\right)$ is the quasicentral in $G / Z(p \infty)$;

7. $A=\left(\left(\left(\left(Z\left(2^{\infty}\right) \cdot B\right) \lambda Q_{8}\right) \times D \cdot B\right) \lambda Q_{8}\right) \times D$, where $Z\left(2^{\infty}\right) \cdot B . Q_{8}$ is the Sylow 2- subgroup of $G, D$ is the finite abelian group,
$Z\left(2^{\infty}\right) \leq C\left(Z\left(2^{\infty}\right) \cdot B\right),\left[Z\left(2^{\infty}\right), Q_{8}\right]=Z\left(2^{\infty}\right)$, the finite group $B$ has a normal series:

$Z\left(2^{\infty}\right) \cdot B=\left\langle B^{\prime},\left[\begin{array}{ll}B, & Q_{8}\end{array}\right]\right\rangle \quad=B_{0} \quad\left\langle\quad B_{1}<\ldots<B_{i}<\ldots<B_{n,} \quad n \geq 1\right.$, $\left.B_{i}=B_{i-1}\right) \lambda\left\langle b_{i}\right\rangle$, for all $i \geq 1,\left|b_{i}\right|=2,\left|B_{0}\right|=2$ and $A / Z(p \infty)$ is the quasicentral in $G / Z(p \infty)$;

8. $A=\left(\left(Z\left(2^{\infty}\right) . B\right) . H\right) \times D$, where $Z\left(2^{\infty}\right) . B . H$ is the Sylow 2-subgroup of $G, D$ is the finite abelian group, $Z\left(2^{\infty}\right) \leq$ $\leq C\left(Z\left(2^{\infty}\right) \cdot B\right),\left[Z\left(2^{\infty}\right), H\right]=Z\left(2^{\infty}\right), H=\langle a\rangle \lambda\langle b\rangle,|a|=|b|=$ $=4,[a, b]=a^{2}$, the finite group $B$ has a normal series:

$Z\left(2^{\infty}\right) \cap B=\left\langle B^{\prime}, \quad[B, H]\right\rangle=B_{0}<B_{1}<\ldots<B_{i}<\ldots<B_{n^{\prime}} \quad n \geq 1$, $B_{i}=B_{i-1} \lambda\left\langle b_{i}\right\rangle$,for all $i \geq 1,\left|b_{1}\right|=2,\left|B_{0}\right|=2,\left(Z\left(2^{\infty}\right) \cdot B\right) \cap H=$ $=Z\left(2^{\infty}\right) \cap H=\left\langle a^{2}, b^{2}\right\rangle$, and $A / Z(p \infty)$ is a quasicentral in $G / Z(p \infty)$.

Proof. Let $G$ be the locally graded $I A N$ group with the infinite non-quasicentral nodal subgroup $A$ of $G$. By Theorem $1 A$ is an almost quasicyclic group containing the finite subgroups that are not normal in $G,|A: A \cap G|<\infty$, and $G / A$ is the Dedekind group. The above mentioned implies that A contains a subgroup $Z(p \infty)$ that is normal in $G, A / Z(p \infty)$ is the finite Dedekind group and furthermore $Z(p \infty) \leq B \leq A$. Pursuant to Definition 1 the group $B$ is the infinite subgroup of $G$. Admittedly, $B$ is normal in $G$ and the factor group $A / Z\left(p^{\infty}\right)$ is the quasicentral subgroup of $G / Z(p \infty)$. According to Theorem 3.1 [8] the subgroup $A$ satisfies the conditions of this theorem. Evidently, $A$ is the group of one of types 1 to 8 of this theorem.

If $A$ is a group of the type 1 of Theorem 3.1 [8], then $A$ is the Dedekind group, $A=Z(p \infty) \times D$ where $D$ is the finite Dedekind group, $p=2$, and $D^{\prime}=\langle e\rangle$. By Lemma $6 p|| D \mid$, the subgroup $A$ contains element $a$ so that a subgroup $\langle a\rangle$ is not normal $p$-subgroup in $\mathrm{G}$. Thus $\mathrm{A}$ is of the type 1 of this theorem.

If $A$ is a group of one of the types $2-8$ of Theorem 3.1 [8], then $A$ is a subgroup of one of the types $2-8$ of this theorem .

Conversely. If $G$ is a group with the normal subgroup $A$ of one of the types $1-8$ of this theorem, then $G / A$ is the Dedekind group. $G$ is evidently the locally graded group. Because $\mathrm{G} / \mathrm{A}$ is the Dedekind group and $A / Z(p \infty)$ is the quasicentral subgroup of $G / Z(p \infty)$, then any infinite subgroup contained in $A$ and any subgroup which contains a subgroup $A$ is normal in $G$. Thus $G$ is the $I A N$ group.

Let $A$ be an infinite subgroup of $G$. If the subgroup $A$ is of the type 1 of this theorem, then the subgroup $A$ contains a subgroup <a that is not normal in $G$. Thus the subgroup $A$ is nonquasicentral subgroup of $G$.

Thus the quasicentral subgroups of the group $G$ are the Dedekind groups, which implies $A$ is a group of one of the types $2-8$ of this theorem. Thus $A$ is the non-Dedekind group, which implies that the subgroup $A$ of one of the types $2-8$ is a nonquasicentral subgroup of $G$. 


\section{Theorem 4.}

The group $G$ is the IANA group with an infinite nonquasicentral nodal subgroup $A$, if and only if $A=Z(p \infty) \times D$, where $D$ is a finite group, $p|| D \mid$, the subgroup A contains an element $a$ so that $\langle a\rangle$-subgroup is not normal of $G$, and $A / Z(p \infty)$ is the quasicentral in $G / Z(p \infty)$.

Proof. Let $G$ be the locally graded $I A N$ group with the infinite non-quasicentral nodal subgroup $A$ of $G$, and $A^{\prime}=\langle p\rangle$. Because $G / A$ is the Dedekind group, $A=Z(p) \times D$, where $D$ is the finite abelian group, $A$ contains the finite subgroups that are not normal in $G$, and $G / A$ is the Dedekind group. The group $G$ is evidently the locally graded $I A N$ group with the nodal subgroup $A$ of the type 1 of Theorem 2, $\mathrm{p}|| D \mid$, the subgroup $A$ contains an element $a$ so that $\langle a\rangle p$-subgroup is not normal in $G$, and $A / Z(p \infty)$ is the quasicentral in $G / Z(p \infty)$.

Let $G$ be a group, $A$ is a subgroup $A$ of $G$, and $A=Z(p \infty) \times D$, where $D$ is a finite group, $p|| D \mid$, the subgroup $A$ contains an element a so that $\langle a\rangle p$-subgroup is not normal in $G$, and $A / Z(p \infty)$ is the quasicentral in $G / Z(p \infty)$.

Conversely. Suppose that $A \unlhd G$ where $A$ is an almost quasicyclic group. Since $A / Z(p \infty)$ is a quasicentral in $G / Z(p \infty)$, then $B / Z(p \infty) \unlhd G / Z\left(p^{\infty}\right)$ for all $B / Z\left(p^{\infty}\right)<A / Z\left(p^{\infty}\right)$. Hence

$\mathrm{B} \unlhd \mathrm{G}, A$ is the abelian subgroup, every infinite subgroup of $A$ and every infinite subgroup of $G$ containing $A$ is a normal subgroup of $G$. By Definition 1 the group $G$ is the IANA group. Hence the subgroup $A$ contains the subgroup that is not normal in $G$, then $A$ is the non-quasicentral in $G$.

\section{References}

[1] KUROSH, A. G.: The Theory of Groups (2 vols.), New York : Chelsea Publishing Comp., 1969.

[2] CHERNIKOV, S. N.: The Groups with the Given Properties of the System of their Subgroups (in Russian), Moskva : Nauka, 1980.

[3] SUBOTTIN, I. J.: The Infinite Groups Generated by the Finite Set in which Every Commutator Subgroup is Invariant (in Russian), Ukr. Mat. zur., vol. 27, No. 3, 1975.

[4] TOMANEK, L., TOMANKOVA, A.: On One Class of the Infinite Non-abelian Groups, Communications - Scientific Letters of the University of Zilina, vol. 12, No. 3, 2010, 44-47, ISSN 1335-4205.

[5] TOMANEK, L.: Groups, Rings and Vector Spaces (in Slovak), EDIS : University of Zilina, 2013, ISBN 978-80-554-0782-1.

[6] HUNGERFORD, T. W.: Algebra, Springer Science + Business Media, LLC, 1974.

[7] HALL, M.: The Theory of Groups. New York : The Macmillan Campany,1959.

[8] KUZENNYJ, N. F., SUBOTTIN, I. J., TOMANEK, L.: About Some Extensions of the Quasicyclic Groups (in Russian), Zbornik Ped. fak. v Presove, UPJS Kosice, vol. XXIV, No. 1, 1990. 